\title{
Global particle-in-cell simulations of Alfvénic modes
}

\author{
A. Mishchenko*, R. Hatzky ${ }^{\dagger}$ and A. Könies* \\ *Max-Planck-Institut für Plasmaphysik, EURATOM-Association, D-17491 Greifswald, Germany \\ ${ }^{\dagger}$ Rechenzentrum der Max-Planck-Gesellschaft und des Max-Planck-Instituts für Plasmaphysik, \\ EURATOM-Association, D-85748 Garching, Germany
}

\begin{abstract}
Global linear gyro-kinetic particle-in-cell (PIC) simulations of electromagnetic modes in pinch and tokamak geometries are reported. The Toroidal Alfvén Eigenmode and the Kinetic Ballooning Mode have been simulated. All plasma species have been treated kinetically (i.e. no hybrid fluid-kinetic or reduced-kinetic model has been applied). The main intention of the paper is to demonstrate that the global Alfvén modes can be treated with the gyro-kinetic PIC method.
\end{abstract}

Keywords: Gyrokinetics, particle-in-cell method

PACS: 01.30.Cc, 52.30.Gz, 52.65.Rr

\section{INTRODUCTION}

The most rigorous first-principle approach to the kinetic theory of MHD modes is the global gyro-kinetic description. Recently, a global gyro-kinetic eigenvalue code $[1,2]$ has been developed. Being capable of capturing all kinetic effects in the linear regime, this approach is however difficult to extend for nonlinear problems. In contrast, the gyrokinetic particle-in-cell (PIC) method can be used both in linear and nonlinear regimes. In the past, electromagnetic PIC simulations have wrestled with stringent numerical constraints associated with the so-called cancellation problem [3, 4]. This problem has been solved recently [3-7]. The key point to its solution is a careful balance between the adiabatic current computed with the markers and the so-called skin terms in Ampére's law discretized on the spatial grid. In this paper, we employ the method of Ref. [7] to solve the cancellation problem.

We use the linear two-dimensional $\delta f$ PIC-code GYGLES [4-11]. The code allows for electromagnetic perturbations and treats all particle species (ions and electrons) on the same footing (kinetically). The code solves the gyro-kinetic Vlasov-Maxwell system of equations $[12,13]$. The distribution function is split into the background part and the perturbation $f_{s}=F_{0 s}+\delta f_{s}$ (the index $s=i, e$ is used for the particle species). The background distribution function is usually taken to be a Maxwellian. The perturbed part of the distribution function is discretized with markers:

$$
\delta f_{s}\left(\mathbf{R}, v_{\|}, \mu, t\right)=\sum_{v=1}^{N_{p}} w_{s v}(t) \delta\left(\mathbf{R}-\mathbf{R}_{v}\right) \boldsymbol{\delta}\left(v_{\|}-v_{v \|}\right) \boldsymbol{\delta}\left(\mu-\mu_{v}\right)
$$

where $N_{p}$ is the number of markers, $\left(\mathbf{R}_{v}, v_{v \|}, \mu_{v}\right)$ are the marker phase space coordinates and $w_{s v}$ is the weight of a marker. The electrostatic and magnetic potentials are 


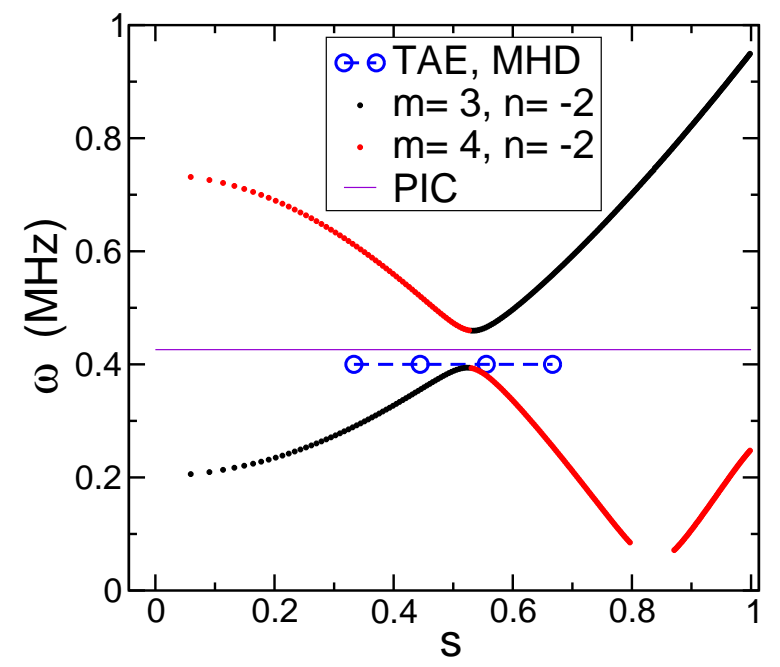

FIGURE 1. The shear Alfvén spectrum in a tokamak. The TAE mode frequency resulting from the PIC simulations is compared with the MHD result. The continuum branches corresponding to the coupling modes with the poloidal mode numbers $m=3$ and $m=4$ are plotted as well. The toroidal mode number is $n=-2$.

discretized with the finite-element method (Ritz-Galerkin scheme):

$$
\phi(\mathbf{x})=\sum_{l=1}^{N_{s}} \phi_{l} \Lambda_{l}(\mathbf{x}), \quad A_{\|}(\mathbf{x})=\sum_{l=1}^{N_{s}} a_{l} \Lambda_{l}(\mathbf{x})
$$

where $\Lambda_{l}(\mathbf{x})$ are the finite elements (tensor product of B splines $\left.[14,15]\right), N_{s}$ is the total number of the finite elements, $\phi_{l}$ and $a_{l}$ are the spline coefficients. A detailed description of the discretization procedure can be found in Refs. $[4,5,7,8,10]$. We apply the socalled phase factor transform [8] to all perturbed quantities in the code. The cancellation problem [3, 4] is solved using the iterative scheme No. 2 described in Ref. [7].

\section{SIMULATIONS}

Toroidal Alfvén Eigenmode (TAE). We consider a large-aspect-ratio circular crosssection tokamak with the major radius $R_{0}=10 \mathrm{~m}$, the minor radius $r_{\mathrm{a}}=1 \mathrm{~m}$, the magnetic field on the axis $B_{0}=0.95 \mathrm{~T}$, the safety factor $q(r)=1.6+0.6\left(r / r_{\mathrm{a}}\right)^{2}$ with $r$ the small radius. The ion and electron temperature and density profiles are taken to be constant with $T_{i}=T_{e}=3.8 \mathrm{keV}$ and $n_{0}=2 \times 10^{18} \mathrm{~m}^{-3}$ [these parameters correspond to $\beta=2 \mu_{0} n_{0}\left(T_{i}+T_{e}\right) / B^{2} \approx 0.7 \%$ ]. We use flat profiles in order to exclude the influence of diamagnetic effects on the TAE mode (their role will be studied elsewhere).

We present the results of our TAE simulations in Figs. 1-3. In Fig. 1, the part of the shear Alfvén spectrum containing the TAE gap is plotted. The mode frequency $\omega=418 \mathrm{kHz}$ lies inside the gap and close to the TAE frequency calculated from ideal MHD theory (CAS3D). The eigenmode structure is shown in Fig. 2 (electrostatic and magnetic potential). The position of the maximum of the potential coincides with 

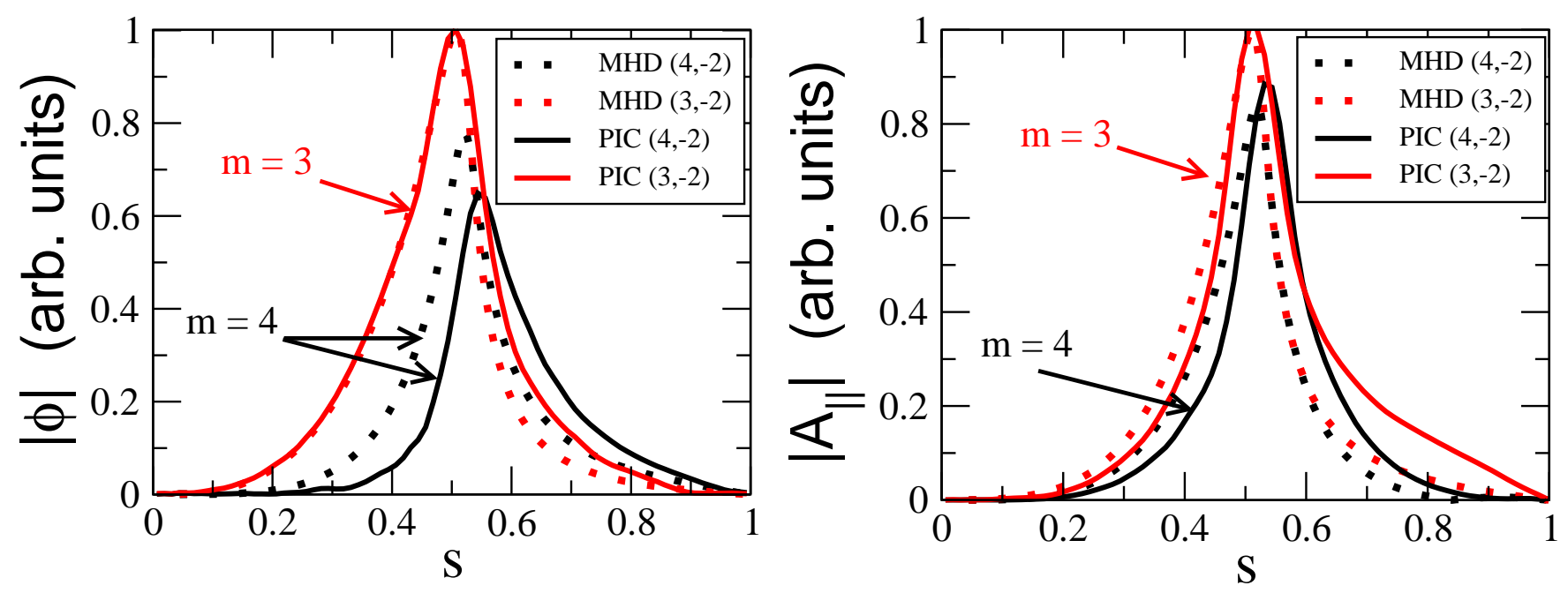

FIGURE 2. The eigenmode structure (the electrostatic and magnetic potentials in arbitrary units) of the TAE mode in a tokamak. One sees two coupling modes with the poloidal mode numbers $m=3$ and $m=4$. The toroidal mode number is $n=-2$. Gyrokinetic PIC result (solid lines) is compared vs. the MHD result (dotted lines).

the position of the gap $r_{m}$ in the shear Alfvén spectrum. Furthermore, the eigenmode structure calculated within ideal MHD is sufficiently close to the gyro-kinetic one. In Fig. 3, the time evolution of the electrostatic and magnetic potential is presented. One sees a decaying mode with two dominant coupled Fourier harmonics with the poloidal mode numbers $m=3$ and $m=4$. Also, the side bands with $m=2$ and $m=5$ having a much smaller amplitude can be seen. The damping rate $\gamma=-3.4 \mathrm{kHz}$ corresponds to $\gamma / \omega=0.8 \%$. The numerical parameters in our simulations are as follows: the number of ion markers $N_{i}=4000000$, the number of electron markers $N_{e}=16000000$, the number of radial B-splines $N_{r}=80$, the number of B-splines in the toroidal direction $N_{z}=32$, the time step $\Delta t=10^{-8} \mathrm{~s}$.

Kinetic Ballooning mode (KBM). In Ref. [16], it has been shown that the lowfrequency part of the Alfvén continuum can become unstable in the presence of the ion temperature gradient. The Alfvén Ion Temperature Gradient driven modes (or Kinetic Ballooning modes) are the drift-Alfvén eigenmodes, destabilized by the free energy of the thermal particles in the presence of the pressure gradient, resulting from the discretization of the unstable continuum due to non-ideal effects (e.g. Finite Larmor Radius effects). These instabilities may have significant implications for both energetic and thermal particle transport.

We consider a circular cross-section tokamak with the major radius $R_{0}=2.0 \mathrm{~m}$, the minor radius $r_{\mathrm{a}}=0.5 \mathrm{~m}$, the safety factor $q(\rho)=1.25+0.67 \rho^{2}+2.38 \rho^{3}-0.06 \rho^{4}$ where $\rho=r / r_{\mathrm{a}}, r$ is the small radius. The temperature profiles $T_{i}(\rho)=T_{e}(\rho)=$ $T_{0} \exp \{-0.5 \tanh [(\rho-0.6) / 0.2]\}$ with the temperature on the axis $T_{0}=7.5 \mathrm{keV}$. The density profile is defined as $n_{0}(\rho)=n_{a} \exp \{-0.44 \tanh [(\rho-0.6) / 0.35]\}$ with the density on the axis $n_{a}$ chosen appropriately in order to obtain the $\beta$-value needed. We choose the toroidal mode number $n=7$ and the poloidal mode numbers $8 \leq m \leq 20$. Results of our simulations are presented in Figs. 4 and 5. In Fig. 4, the frequency and 

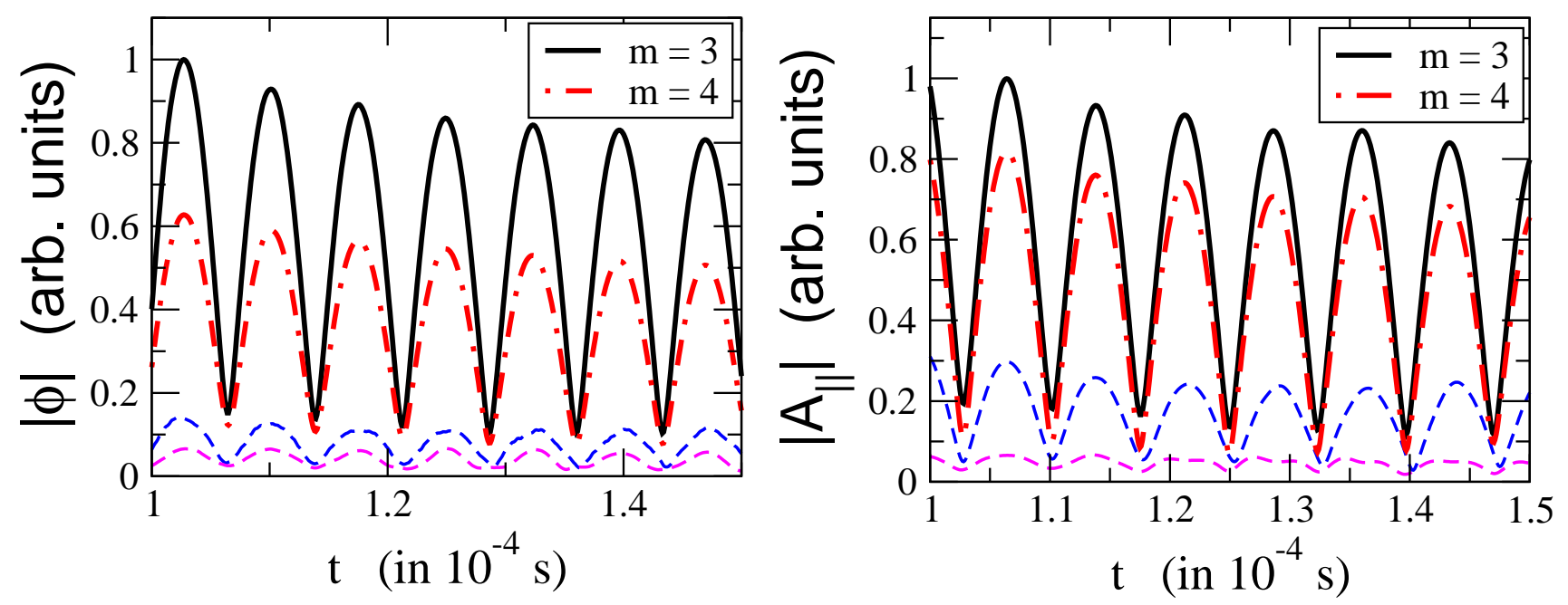

FIGURE 3. Time evolution of the electrostatic and magnetic potentials (arbitrary units). The TAE mode in a tokamak. One sees that the modes with the poloidal mode numbers $m=3$ and $m=4$ couple with each other. The toroidal mode number is $n=-2$.

the growth rate of the instability is plotted. One can see how the electrostatic mode (here, the Trapped Electron Mode coexisting with the Ion Temperature Gradient driven mode) is replaced by the KBM instability when $\beta$ is large enough (recall that the time evolution particle-in-cell approach delivers the most unstable mode in the spectrum). In Fig. 5, the spatial structure of the electrostatic and the magnetic potentials is shown in the tokamak cross-section. One can see that both potentials have ballooning structure. The numerical parameters in our simulations are as follows: the number of the ion markers $N_{i}=1000000$, the number of the electron markers $N_{e}=4000000$, the number of radial B-splines $N_{r}=64$, the number of B-splines in the toroidal direction $N_{z}=32$, the time step $\Delta t=5 \times 10^{-9} \mathrm{~s}$.

\section{CONCLUSIONS}

In this paper, we have presented global gyrokinetic PIC simulations of the Toroidal Alfvén Eigenmode and the Kinetic Ballooning Modes. The key numerical component of our simulations is the solution of the cancellation problem $[3,4]$ using the iterative approach of Ref. [7]. This allows to keep the number of markers in the same range as in comparable electrostatic PIC simulations. The main result of our paper is to demonstrate that global Alfvén modes can be treated in a unified manner with the gyrokinetic PIC method. Up to now, the kinetic properties of the global Alfvén modes in a tokamak geometry have been approached using reduced-kinetic [17], hybrid fluid-kinetic [18] or gyrokinetic-eigenvalue approaches [1]. The drawback of the reduced-kinetic and hybrid fluid-kinetic approaches is that they can not guarantee to include all relevant physical mechanisms. The gyrokinetic eigenvalue approach being capable to capture all physics is limited to linear problems only. Being a first-principle non-perturbative approach, the gyrokinetic PIC simulations can recover all kinetic effects, too. In contrast with the 

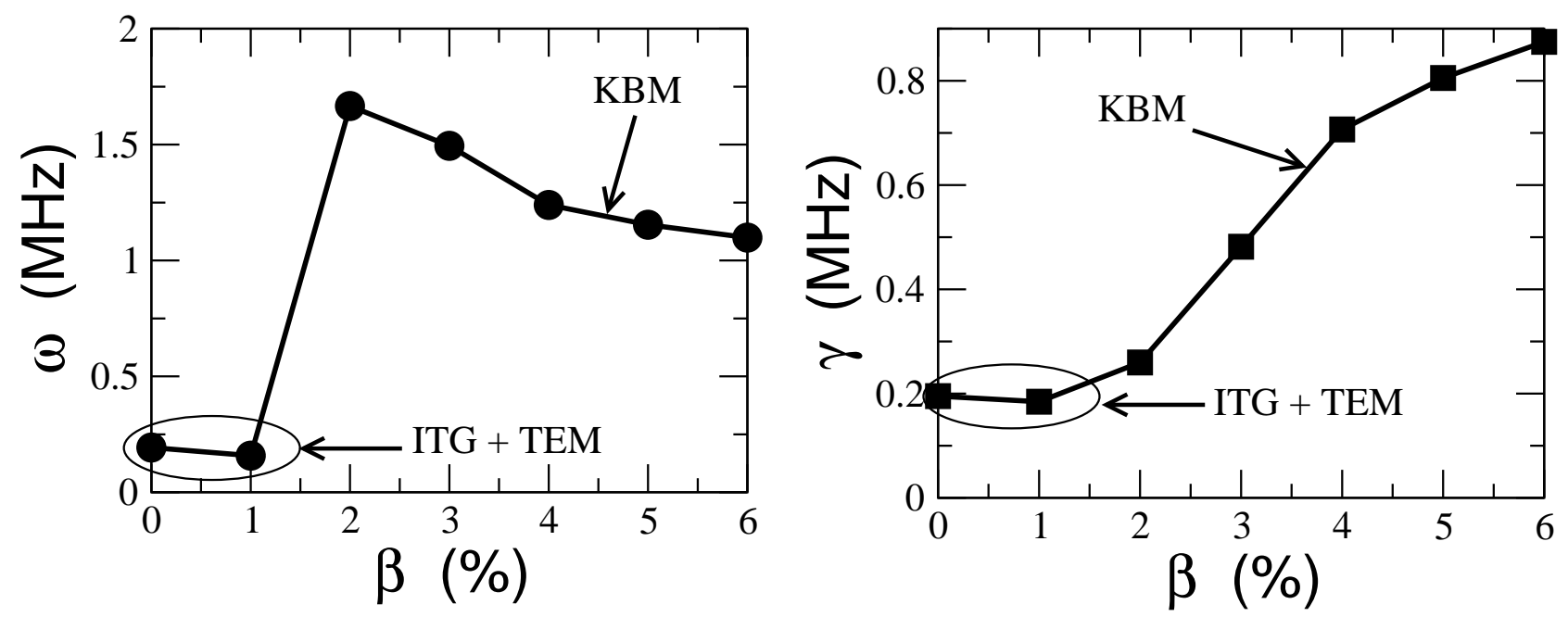

FIGURE 4. The frequency and the growth rate of the ITG+TEM (small $\beta$ ) and KBM (larger $\beta$ ) instabilities in tokamak geometry.
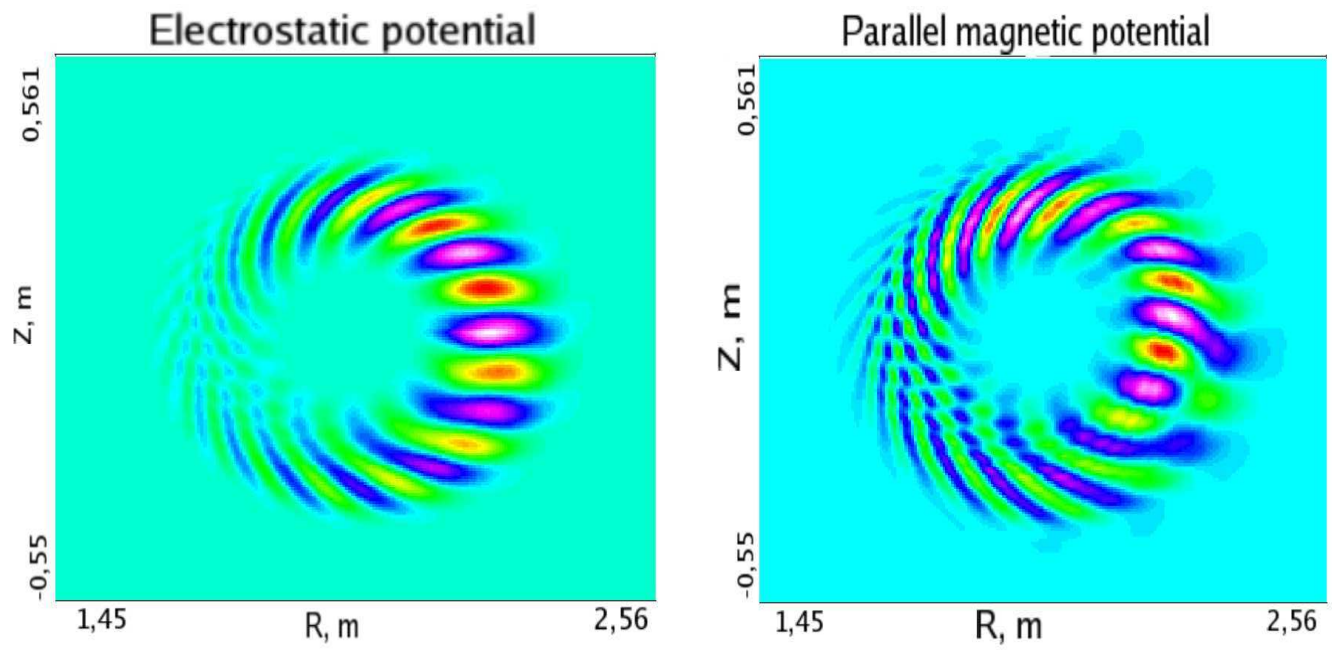

FIGURE 5. The structure of electrostatic and magnetic potentials (KBM instability) in the tokamak cross-section. Parameters as in Fig. 4 corresponding to the point with $\beta=4 \%$.

eigenvalue approach, the PIC method can be easily extended to the nonlinear regimes. The linear simulations presented in this paper are an important step towards the global nonlinear gyrokinetic treatment of electromagnetic modes in tokamak geometry (both electromagnetic turbulence and nonlinear dynamics of kinetic MHD modes destabilized by the fast particles). The PIC simulation of the kinetic effects caused by the fast particles will be the focus of our future work.

\section{ACKNOWLEDGMENTS}

We acknowledge the support of J. Nührenberg for this work and many discussions 
with him. We appreciate many discussions with C. Nührenberg. Furthermore we thank P. Helander for helpful comments and carefully reading the manuscript. On the computational side we have to thank H. Leyh and M. Borchardt.

\section{REFERENCES}

1. P. Lauber, S. Günter, and S. D. Pinches, Phys. Plasmas 12, 122501 (2005).

2. P. Lauber, S. Günter, A. Könies, and S. D. Pinches, J. Comp. Phys. 226, 447 (2007).

3. Y. Chen and S. Parker, J. Comp. Phys 189, 463 (2003).

4. A. Mishchenko, R. Hatzky, and A. Könies, Phys. Plasmas 11, 5480 (2004).

5. A. Mishchenko, A. Könies, and R. Hatzky, in Proc. of the Joint Varenna-Lausanne International Workshop (Società Italiana di Fisica, Bologna, 2004).

6. R. Hatzky, A. Könies, and A. Mishchenko, in Proc. of the Joint Varenna-Lausanne International Workshop (Società Italiana di Fisica, Bologna, 2004).

7. R. Hatzky, A. Könies, and A. Mishchenko, J. Comp. Phys. 225, 568 (2007).

8. M. Fivaz, S. Brunner, G. de Ridder, O. Sauter, T. M. Tran, J. Vaclavik, L. Villard, and K. Appert, Comp. Phys. Commun. 111, 27 (1998).

9. S. Sorge and R. Hatzky, Plasma Phys. Control. Fusion 44, 2471 (2002).

10. A. Mishchenko, A. Könies, and R. Hatzky, Phys. Plasmas 12, 062305 (2005).

11. A. Mishchenko, R. Hatzky, and A. Könies, in Proc. of the Joint Varenna-Lausanne International Workshop (AIP Conference Proceedings, New York, 2006).

12. T. S. Hahm, Phys. Fluids 31, 2670 (1988).

13. T. S. Hahm, W. W. Lee, and A. J. Brizard, Phys. Fluids 31, 1940 (1988).

14. C. de Boor, A Practical Guide to Splines (Springer-Verlag, New York, 1978).

15. K. Höllig, Finite Element Methods with B-Splines (Society for Industrial and Applied Mathematics, Philadelphia, 2003).

16. F. Zonca, L. Chen, and R. Santoro, Plasma Phys. Controlled Fusion 38, 2011 (1996).

17. G. Y. Fu, H. L. Berk, and A. Pletzer, Phys. Plasmas 12, 082505 (2005).

18. Y. Nishimura, Z. Lin, and W. X. Wang, Phys. Plasmas 14, 042504 (2007). 